\title{
SIMULAÇÃO E OTIMIZAÇÃO DA PURIFICAÇÃO DO PRODUTO OBTIDO A PARTIR DO CRAQUEAMENTO DE ÓLEO VEGETAL EMPREGANDO O ASPEN HYSYS.
}

\author{
E. C. $\operatorname{COSTA}^{1}$, A. K. TAKAKURA ${ }^{1}$, N. T. MACHADO ${ }^{1}$. \\ ${ }^{1}$ Universidade Federal do Pará, Programa de Pós-Graduação em Engenharia de Recursos Naturais da \\ Amazônia \\ E-mail para contato: elineia_castro@yahoo.com.br
}

\begin{abstract}
RESUMO - Este trabalho tem como objetivo realizar simulações empregando o simulador comercial Aspen Hysys para avaliar os parâmetros operacionais ótimos à separação de componentes indesejáveis em uma mistura de hidrocarbonetos na faixa de querosene $\left(175-235^{\circ} \mathrm{C}\right)$. A composição da mistura utilizada nas simulações foi retirada de dados da literatura obtidos experimentalmente a partir de uma unidade de craqueamento onde se utilizou óleo de palma para obter biocombustível. Para descrever o equilíbrio de fases do sistema multicomponente foram aplicados modelos de coeficiente de atividade (Wilson, NRTL e UNIQUAC) e equações de estado (PR, SRK e TST) com a matriz de parâmetros de interação, entre os constituintes da mistura multicomponente, da base de parâmetros do simulador. Mantendo-se constantes as condições de operação e a composição da corrente de entrada da coluna de destilação, se avaliou quais métodos de propriedades termodinâmicas forneceram os melhores resultados para o processo de purificação.
\end{abstract}

\section{INTRODUÇÃO}

Além da crescente preocupação com o meio ambiente no que tange à proteção e conservação ambiental, existe ainda a perspectiva de esgotamento das reservas de origem fóssil nas próximas décadas. Neste contexto, vários pesquisadores vêm se concentrando em fontes renováveis de combustíveis líquidos para substituir ou diminuir o consumo de produtos fósseis no futuro, com destaque para derivados da biomassa, em razão da sua natureza renovável, ampla disponibilidade e, biodegradabilidade. Dentre as diversas rotas tecnológicas investigadas, se destaca o craqueamento. No processo clássico de craqueamento de óleos vegetais, os triglicerídeos são transformados em moléculas de estrutura simples através do aquecimento a temperaturas acima de $350{ }^{\circ} \mathrm{C}$, normalmente sob a ação de catalisadores. Os produtos obtidos são constituídos de carbono e hidrogênio, com frações nas faixas do diesel, gasolina, e querosene. Muitos estudos são realizados visando melhorar as propriedades dos combustíveis produzidos pelo processo de craqueamento a partir de diferentes matérias-primas derivadas da biomassa (Lhamas, 2013). Desta forma, o uso de simuladores de processos se mostra de extrema importância, pois tais ferramentas são capazes de descrever o comportamento de processos industriais (dentre eles a purificação de biocombustíveis) com aceitável precisão, possibilitando e contribuindo para a otimização desses processos. 


\section{9 a 22 de outubro de 2014 \\ Florianópolis/SC}

Neste trabalho utiliza-se uma mistura obtida a partir do craqueamento de óleo vegetal com faixa de hidrocarbonetos similar ao querosene de petróleo. Segundo Farah (2012) o QAV-1 (querosene de aviação) apresenta predominância de hidrocarbonetos parafínicos, compostos mais desejáveis para se obter chamas limpas e sem fuligem, contendo também hidrocarbonetos aromáticos que apresentam as piores características de combustão, pois, provocam aumento da radiação na câmara de combustão, assim como a formação de fuligem, sendo necessária sua remoção. Quanto à presença de oxigenados, estes favorecem o aumento da acidez, corrosividade, formação de gomas e odor, sendo também, nesse caso, necessária a remoção de tais compostos (Lhamas, 2013).

Este trabalho teve como objetivo simular a destilação de produto obtido por craqueamento similar ao querosene de petróleo, utilizando o simulador comercial Aspen Hysys. Foram avaliadas se ocorrem diferenças significativas nas simulações quando utilizados diferentes pacotes termodinâmicos disponíveis no simulador.

\section{SIMULAÇÃO DE PROCESSOS}

\subsection{Modelos Termodinâmicos}

O sucesso da modelagem de processos depende da precisão da descrição das propriedades termodinâmicas e das condições de equilíbrio de fases. O simulador Aspen Hysys possui diversas opções de modelos termodinâmicos para descrever o equilíbrio de fases da maioria dos processos aplicados na indústria química. Estes modelos termodinâmicos pretendem interpretar e predizer o comportamento físico-químico da matéria em equilíbrio (Takakura, 2009). O cálculo do equilíbrio de fases pode ser baseado na aplicação de equações de estado (EDE). Assim, uma única equação é usada para representar todas as fases fluidas (Teribeli, et al. 2006). As equações de estado cúbicas de SoaveRedlich-Kwong (SRK) e Peng-Robinson (PR) e suas modificações são as mais amplamente utilizadas na indústria, no entanto, existem outras equações que podem ser utilizadas para descrever o equilíbrio de fases de uma mistura e, portanto, para descrever processos. Entre elas citamos a equação de TwuSim-Tassone (Twu et al, 2002).

A Equação 1 representa a forma geral de uma equação de estado cúbica.

$$
P=\frac{R T}{v-b}-\frac{a}{(v+u b)(v+w b)}
$$

As constantes, $u$ e $w$ dependem da EDE. Twu et al (2002) propôs uma metodologia para encontrar uma EDE cúbica para prever a densidade líquida para hidrocarbonetos pesados e compostos polares de forma mais precisa que PR e SRK. Para a equação TST, $u=-0.5$ e $w=3.0$. Para a equação SRK, $u=0, w=1$. Para PR, $u=-0.4142$ and $w=2.4141$. SRK é a melhor escolha quando se trabalha com metano e PR é melhor de $n$-pentano até n-heptano; e TST é superior para $n$-octano e compostos com números de carbono mais elevados, assim como, para componentes polares (Twu et al, 2002).

O Aspen Hysys também fornece outro de método de cálculo de propriedades, através de 
modelos termodinâmicos para descrever a não idealidade da fase líquida, baseados no coeficiente de atividade. Dentre os vários modelos disponíveis no Aspen Hysys, neste trabalho foram utilizados os modelos NRTL, UNIQUAQ e Wilson para descrever a não idealidade da fase líquida, sendo considerada a idealidade para a fase vapor. Estes modelos empregam a teoria de composição local e apresentam grande aplicação prática na determinação do equilíbrio líquido-vapor. Os modelos de composição local possuem flexibilidade limitada no ajuste de dados experimentais, porém, são adequados para uma vasta gama de problemas de engenharia (Meireles e Pereira, 2013).

A equação de Wilson (1964) se mostra útil para sistemas com grandes desvios da idealidade e multicomponentes. Para sistemas multicomponentes a equação está representada a seguir:

$$
\begin{aligned}
& \ln \gamma_{k}=1-\ln \left(\sum_{i} x_{i} \Lambda_{k i}\right)-\sum_{j} \frac{x_{j} \Lambda_{j k}}{\sum_{i} x_{i} \Lambda_{j i}} \\
& \text { Onde: } \quad \Lambda_{\mathrm{ij}}=\frac{\mathrm{v}_{\mathrm{i}}}{\mathrm{V}_{\mathrm{j}}} \exp \left(-\frac{\mathrm{A}_{\mathrm{ij}}}{\mathrm{T}}\right) \quad \text { e } \quad \Lambda_{i i}=\Lambda_{j j}=1
\end{aligned}
$$

O modelo termodinâmico molecular NRTL (non-random two-liquids), proposto por Renon e Prausnitz (1968), é uma extensão da equação de Wilson e utiliza a mecânica estatística aliada ao conceito de composição local para representar a estrutura líquida. A diferença para o modelo de Wilson é que o modelo NRTL é aplicável a sistemas com miscibilidade parcial, o que permite representar os equilíbrios liquido-vapor, liquido-liquido e liquido-liquido-vapor (Santana, 2008). A equação do modelo NRTL para o coeficiente de atividade está representada a seguir:

$$
\begin{aligned}
& \ln \gamma_{i}=\frac{\sum_{j} \tau_{j i} G_{j i} x_{j}}{\sum_{j} G_{j i} x_{j}}+\sum_{j} \frac{x_{j} G_{i j}}{\sum_{k} G_{k j} x_{k}}\left[\tau_{i j}-\frac{\sum_{k} x_{k} \tau_{k j} G_{k j}}{\sum_{k} G_{k j} x_{k}}\right] \\
& \tau_{j i}=\frac{A_{i j}}{T}, \quad G_{i j}=\exp \left(-\alpha_{i j} \tau_{i j}\right), \quad \tau_{i i}=\tau_{j j}=0, \quad G_{i i}=G_{j j}=1
\end{aligned}
$$

Onde: $\quad \gamma=$ coeficiente de atividade do componente $\mathrm{i} ; \mathrm{x}_{i}=$ fração molar do componente $\mathrm{i} ; \mathrm{T}=$ temperatura; $\mathrm{A}_{i j}=$ parâmetro de interação entre os componentes $\mathrm{i}$ e $\mathrm{j}, \alpha_{i j}=$ representa a não aleatoriedade da mistura, ou seja, supõe que os componentes na mistura não se distribuem aleatoriamente e seguem um padrão ditado pela composição local.

Neste trabalho, também foi utilizado o modelo UNIQUAC (Universal Quase Chemical) proposto por Abrams e Prausnitz (1975) e que utiliza a mecânica estatística e a teoria quase química de Guggenhein (1952). Essa equação consiste de duas partes: uma parte combinatorial, que descreve as contribuições entrópicas dos componentes, e uma parte residual, que expressa as forças intermoleculares que são responsáveis pela entalpia da mistura. A parte combinatorial depende apenas da composição, do tamanho e da forma das moléculas. Essa parte necessita apenas de dados dos componentes puros, no entanto, a parte residual depende das forças intermoleculares, de onde surgem os dois parâmetros ajustáveis (Santana, 2008). O método UNIQUAC em termos de coeficiente de atividade é formado pelo seguinte conjunto de Equações:

$$
\ln \gamma_{i}=\ln \gamma_{i}^{C}+\ln \gamma_{i}^{R}
$$




$$
\begin{aligned}
& \ln \gamma_{i}^{C}=\ln \frac{\Phi_{i}}{x_{i}}+\frac{z}{2} q_{i} \ln \frac{\theta_{i}}{\Phi_{i}}+l_{i} \frac{\Phi_{i}}{x_{i}} \sum_{j} x_{j} l_{j} \\
& \ln \gamma_{i}^{R}=q_{i}\left[1-\ln \left(\sum_{j} \theta_{j} \tau_{j i}\right)-\sum_{k} \frac{\theta_{j} \tau_{i j}}{\theta_{k} \tau_{k j}}\right]
\end{aligned}
$$

Onde:

$\theta_{i}=\frac{q_{j} x_{i}}{\sum_{j} q_{j} x_{j}} \quad \Phi_{i}=\frac{\tau_{j} x_{i}}{\sum_{j} r_{j} x_{j}} \quad l_{i}=\frac{z}{2}\left(r_{i}-q_{i}\right)-\left(r_{i}-1\right) \quad \mathrm{e} \quad z=10$

Nessas equações $x_{i}$ é a fração molar do componente i, $\theta_{i}$ é a fração da área, $\Phi_{i}$ é uma fração similar à fração de volume e z é um número de coordenação. Nas somatórias das equações (5) e (6), estão envolvidos todos os compostos, inclusive o componente i (Terron, 2009).

\subsection{Simulação do Processo de Destilação}

O projeto foi concebido segundo algumas premissas e considerações: a torre de destilação opera com 11 pratos; com condensador total; com razão de refluxo de 0,5; a composição da corrente de alimentação utilizada foi a obtida experimentalmente por Mota (2013); a temperatura da alimentação para todas as plantas é $70{ }^{\circ} \mathrm{C}$ e a alimentação é inserida no sexto prato a partir do topo; a coluna tem capacidade simulada de $100 \mathrm{kmol} / \mathrm{h}$ de mistura e opera à pressão constante de $101,3 \mathrm{kPa}$.

Mota (2013) realizou o craqueamento de óleo de palma e após algumas etapas de separação dos componentes da mistura obtida com o craqueamento, obteve como um dos produtos um biocombustível na faixa de destilação de $175-235^{\circ} \mathrm{C}$. Através da análise desse biocombustível, o autor afirmou que a faixa dos hidrocarbonetos presentes no biocombustível obtido entre $175-235^{\circ} \mathrm{C}$, continha hidrocarbonetos entre $\mathrm{C}_{8}$ a $\mathrm{C}_{17}$, sendo preponderante a faixa de hidrocarbonetos $\mathrm{C}_{11}$ a $\mathrm{C}_{12}$. Desta forma, o autor inferiu que, o biocombustível se aproximava da faixa de hidrocarbonetos presentes no querosene derivado do petróleo. O bio-querosene; obtido por Mota (2013) corresponde a um biocombustível composto de hidrocarbonetos aromáticos, parafínicos normais, naftênicos e olefínicos, além de compostos oxigenados. A Tabela 1 apresenta a composição do biocombustível obtido por Mota (2013) referente ao teor de oxigenados e hidrocarbonetos. Salienta-se que no simulador foi cadastrado cada componente separadamente, porém, visando simplificar a análise da simulação as frações de hidrocarbonetos não aromáticos foram somadas, bem como, as de todos os oxigenados e hidrocarbonetos aromáticos.

As propriedades críticas e fator acêntrico dos componentes da mistura, cadastrados como hipotéticos no simulador, foram estimados pelo método de contribuição de grupos proposto por Constantinou e Gani (1994) e Constantinou et al. (1995).

Tabela 1 - Composição utilizada nas simulações

\section{Componentes}

Hidrocarbonetos Aromáticos

Hidrocarbonetos Não aromáticos

Oxigenados

\section{Porcentagem em massa $(\%)$}

7,04

79,33

13,63 


\section{RESULTADOS E DISCUSSÕES}

Na Tabela 2 estão apresentados os parâmetros de processos, fluxo molar, temperatura e fração molar, para cada corrente envolvida nas simulações utilizando os pacotes termodinâmicos PR, SRK, TST, NRTL, UNIQUAQ e WILSON. Nesta tabela são comparadas as frações em moles dos componentes de interesse no processo aqui estudado (Hidrocarbonetos Aromáticos e Não aromáticos e, Compostos Oxigenados).

Tabela 2 - Parâmetros de processo e fração molar nas correntes envolvidas na simulação.

\begin{tabular}{|c|c|c|c|c|c|c|c|}
\hline & & \multicolumn{6}{|c|}{ Corrente de Topo } \\
\hline & Alimentação & PR & SRK & TST & NRTL & UNIQUAQ & Wilson \\
\hline $\mathrm{T}\left({ }^{\circ} \mathrm{C}\right)$ & 70 & 65,91 & 66,53 & 42,02 & $-9,3987$ & $-25,59$ & 2,3198 \\
\hline Fluxo (kmol/h) & 100 & 41,7999 & 41,8001 & 17,3803 & 17,38 & 8,9990 & 19,7997 \\
\hline A & 0,09458 & 0,2263 & 0,2263 & 0,5880 & 1,0000 & 1,0000 & 0,4777 \\
\hline \multirow[t]{3}{*}{ Fração Molar } & 0,75840 & 0,5166 & 0,5171 & 0,2433 & 0,0000 & 0,0000 & 0,1864 \\
\hline & 0,14702 & 0,2571 & 0,2566 & 0,1686 & 0,0000 & 0,0000 & 0,3359 \\
\hline & & \multicolumn{6}{|c|}{ Corrente de Fundo } \\
\hline $\mathrm{T}\left({ }^{\circ} \mathrm{C}\right)$ & 70 & 210,86 & 210,61 & 203,36 & 186,90 & 183,27 & 201,01 \\
\hline Fluxo (kmol/h) & 100 & 58,200 & 58,200 & 82,620 & 82,621 & 91,001 & 80,200 \\
\hline \multirow{3}{*}{ Fração Molar } & 0,09458 & 0,0000 & 0,0000 & 0,0000 & 0,0028 & 0,0050 & 0,0000 \\
\hline & 0,75840 & 0,8721 & 0,9317 & 0,9538 & 0,9055 & 0,8334 & 0,8996 \\
\hline & 0,14702 & 0,0680 & 0,0683 & 0,0462 & 0,0918 & 0,1616 & 0,1004 \\
\hline
\end{tabular}

*A - Hidrocarbonetos Aromáticos; B - Hidrocarbonetos não aromáticos; C - Compostos Oxigenados.

Foi verificado nas simulações que há diferenças na composição total do vapor das correntes empregando as diferentes equações de estado, bem como, quando se emprega modelos de coeficiente de atividade.

Para as EDE's as diferenças se manifestam entre as equações de PR e SRK comparativamente a TST. Além, das diferenças no perfil de temperatura e fluxo molar (o que pode ser melhor visualizado através das Figuras 1 e 3) também é possível observar as diferenças nas frações molares dos componentes. Observando a Tabela 2, notamos que a EDE de TST é mais eficaz na retirada dos compostos oxigenados. No que se refere aos hidrocarbonetos aromáticos as três EDE's mostraram bons resultados.

Para os modelos de coeficiente de atividade as maiores discrepâncias se manifestaram entre o modelo de Wilson comparativamente aos modelos Uniquaq e Nrtl. Na Figura 2, que exibe o perfil de temperatura ao longo da coluna de destilação, é possível visualizar a diferença entre os modelos. No que tange a retirada dos compostos indesejáveis, os três modelos tiveram bons resultados na retirada de aromáticos e concentração de hidrocarbonetos não aromáticos na corrente de fundo, porém, apenas o modelo de Wilson se mostrou eficaz também na separação de compostos oxigenados, os outros dois modelos não mostraram bom desempenho.

Quanto à análise entre os tipos de modelos utilizados, as diferenças nos resultados são intensas, com exceção das equações TST e Wilson, cujos valores de fluxos e frações molares 
foram próximos, quando comparados aos valores dos demais modelos de coeficiente de atividade.

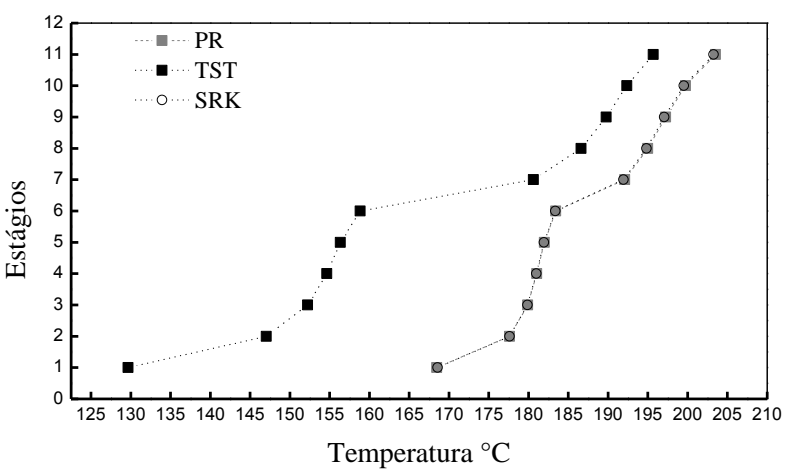

Figura 1 - Temperatura ao Longo dos Estágios da Coluna (EDE's).

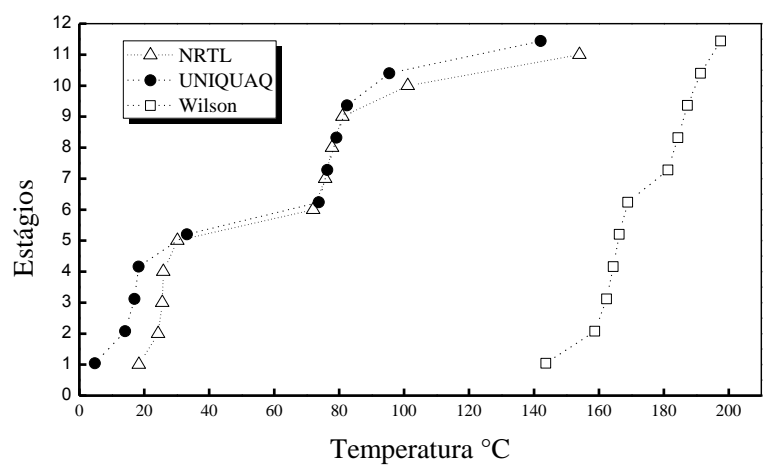

Figura 2 - Temperatura ao Longo dos Estágios da Coluna (Coef. de Atividade).

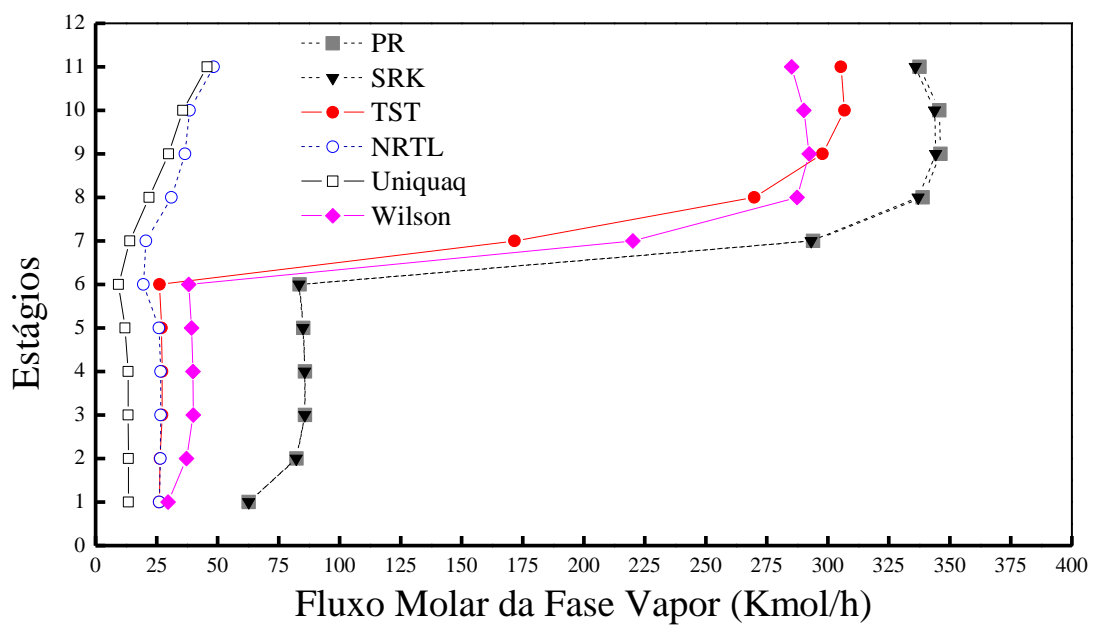

Figura 3 - Fluxo de Vapor ao Longo dos Estágios da Coluna.

\section{CONCLUSÕES}

Este trabalho teve como objetivo simular uma planta simplificada de destilação utilizando o simulador de processos comercial Aspen Hysys, para retirada de compostos oxigenados e hidrocarbonetos aromáticos de uma mistura obtida por craqueamento de óleo vegetal, similar ao querosene de petróleo. Foram avaliadas se ocorrem diferenças significativas nas simulações quando utilizadas diferentes equações de estado (PR, SRK, TST) e modelos de coeficiente de atividade (Wilson, Uniquaq, Nrtl) disponíveis no simulador para o cálculo das propriedades termodinâmicas.

As diferenças entre os modelos de EDE's e coeficientes de atividade são visíveis quando se 
observa e compara os resultados das equações Uniquaq e Nrtl, com as demais. A equação de Wilson obteve desempenho relativamente próximo ao das EDE's, principalmente aos da equação TST, resultando na separação dos compostos indesejáveis da mistura. A equação TST foi a que mostrou melhores resultados no que tange a concentração de hidrocarbonetos não aromáticos na corrente de fundo e separação de compostos oxigenados e hidrocarbonetos aromáticos.

As simulações com as equações PR e SRK obtiveram resultados semelhantes para todas as variáveis estudas. Ambas conseguiram separar os constituintes indesejáveis, o que confirma que estes pacotes termodinâmicos satisfazem este tipo de planta de processo, porém, a equação TST apresenta desempenho superior. Salienta-se que segundo Twu et al,(2002) a equação TST foi proposta para hidrocarbonetos com número de carbonos superior a $\mathrm{C}_{8}$ o que explica os melhores resultados quando comparada a PR e SRK.

\section{REFERÊNCIAS}

ABRAMS, D.S., PRAUSNITZ, J.M. Statistical thermodynamics of liquid mixtures: A new expression for the excess Gibbs energy of eartly or completely miscible substances. AIChE Journal, v. 21, p. $116-128,1975$.

ASPENTECH,Thermodynamics and HYSYS, 2000.

CONSTANTINOU, L.; GANI, R. New group contribution method for estimating properties of pure compounds. American Institute of Chemical Engineers Journal. v. 40, p. 1697-1710, 1994.

CONSTANTINOU, L.; GANI, R.; O'CONNELL, J. P. Estimation of the acentric factor and the liquid molar volume at $298 \mathrm{~K}$ using a new group contribution method. Fluid Phase Equilibria. v. 103, p. 11-22, 1995.

FARAH, M. A. Petróleo e seus derivados: definição, constituição, aplicação, especificações, características de qualidade. Rio de Janeiro: LTC, 2012.

GUGGENHEIM, E.A. The theory of the equilibrium properties. of some simple classes of mixtures solutions and alloys. Clarendon Press, 1952, $270 \mathrm{p}$

LHAMAS, D. E. L. Estudo do Processo de Craqueamento Termocatalítico do Óleo de Palma (Elaeis guineensis) e do Óleo de Buriti (Mauritia flexuosa L.) para Produção de Biocombustível. 2013. 216 f. Tese (Doutorado em Engenharia de Recursos Naturais) - Universidade Federal do Pará. Belém - PA, 2013.

MEIRELES, M. A. A.; PEREIRA, C. G. Fundamentos de Engenharia de Alimentos.São Paulo: Atheneu, 2013.

MOTA, S. A. P.Craqueamento termo-catalítico de óleos vegetais em diferentes escalas de produção. 2013. 332 f. Tese (Doutorado em Engenharia de Recursos Naturais)- Universidade Federal do Pará. Belém, Pará, 2013. 
PENG, D.Y.; ROBINSON, D.B. A New Two-Constant Equation of State. Industrial and Engineering Chemical Fundamental 15: 59-64, 1976.

RENON H. PRAUSNITZ, J.M. Local composition in thermodynamic excess function for liquid mixtures. AICHE Journal. v.14, p. 135-144, 1968.

SANTANA, G. C. de S. Simulação e análise de custos na produção de biodiesel a partir de óleos vegetais. 2008. 175 f. Tese (Doutorado em Engenharia Química) - Universidade Estadual de Campinas. Campinas - SP, 2008.

SOAVE, G. Equilibrium Constants from a Modified Redlich-Kwong Equation of State. Chemical Engineering Science v. 27, p.1192-1203, 1972.

TAKAKURA, A. K. Simulação do Processo Contínuo de Produção e Purificação de Biodiesel de Óleo d Palma Bruto. 2009. 96 f. Dissertação (Mestrado em Engenharia Química) - Universidade Federal do Pará. Belém - PA, 2009.

TERIBELE, T.; SANTOS, J. L.; ARAÚJO, M. E. Simulação De Uma Unidade De Turbo-Expansão De Gás Natural Empregando Diferentes Equações De Estado. In: Cobeq 2006 - XVI Congresso Brasileiro de Engenharia Química e III Congresso Brasileiro de Termodinâmica Aplicada, 2006, Santos.

TERRON, L., R., Termodinâmica Química Aplicada, Manole, $1^{\text {a }}$ Edição, 2009.

TWU, C. H., et. al., "A Versatile Liquid Activity Model for SRK, PR and A New Cubic Equation of State TST," Fluid Phase Equilibria, 194-197, pp. 385-399 (2002). 